\title{
Exploring the roles of integrin binding and cytoskeletal reorganization during mesenchymal stem cell mechanotransduction in soft and stiff hydrogels subjected to dynamic compression
}

\author{
Andrew J. Steward ${ }^{a, b}$, Diane R. Wagner ${ }^{b}$, Daniel J. Kelly ${ }^{a, c, *}$ \\ ${ }^{\mathrm{a}}$ Trinity Centre for Bioengineering, Trinity College Dublin, Dublin 2, Ireland \\ bioengineering Graduate Program, Department of Aerospace and Mechanical Engineering, University of Notre Dame, \\ Notre Dame, IN, United States \\ ${ }^{c}$ Department of Mechanical and Manufacturing Engineering, School of Engineering, Trinity College Dublin, Dublin 2, Ireland
}

\section{A R T I C L E I N F O}

Article history:

Received 3 April 2013

Received in revised form

17 July 2013

Accepted 22 July 2013

Keywords:

Pericellular matrix

Mesenchymal

Mechanobiology

Cytoskeleton

Mechanotransduction

Focal adhesion

\begin{abstract}
A B S T R A C T
The objective of this study was to explore how the response of mesenchymal stem cells (MSCs) to dynamic compression (DC) depends on their pericellular environment and the development of their cytoskeleton. MSCs were first seeded into 3\% agarose hydrogels, stimulated with the chondrogenic growth factor TGF- $\beta 3$ and exposed to DC ( 10\% strain at $1 \mathrm{~Hz}$ ) for $1 \mathrm{~h}$ on either day 7, 14, or 21 of culture. At each time point, the actin, vimentin and tubulin networks of the MSCs were assessed using confocal microscopy. Similar to previous results, MSCs displayed a temporal response to DC; however, no dramatic changes in gross cytoskeletal organization were observed with time in culture. Vinculin (a membrane-cytoskeletal protein in focal adhesions) staining appeared more intense with time in culture. We next aimed to explore how changes to the pericellular environment, independent of the duration of exposure to TGF- $\beta 3$, would influence the response of MSCs to DC. To this end, MSCs were encapsulated into either 'soft' or 'stiff' agarose hydrogels that are known to differentially support pericellular matrix (PCM) development. The application of DC led to greater relative increases in the expression of chondrogenic marker genes in the stiffer hydrogels, where the MSCs were found to have a more well developed PCM. These increases in gene expression were not observed following the addition of RGDS, an integrin blocker, suggesting that integrin binding plays a role in determining the response of MSCs to DC. Microtubule organization in MSCs was found to adapt in response to DC, but this effect was not integrin mediated, as this cytoskeletal reorganization was also observed in the presence of RGDS. In conclusion, although the PCM, integrin binding, and cytoskeletal reorganization are all involved in mechanotransduction of DC, none of these factors in isolation was able to completely explain the temporal mechanosensitivity of MSCs to dynamic compression.
\end{abstract}

(c) 2013 Elsevier Ltd. All rights reserved.

*Corresponding author. Tel.: +353 1 8963947; fax: +353 16795554.

E-mail address: kellyd9@tcd.ie (D.J. Kelly).

1751-6161/\$ - see front matter (c) 2013 Elsevier Ltd. All rights reserved.

http://dx.doi.org/10.1016/j.jmbbm.2013.07.020

Please cite this article as: Steward, A.J., et al., Exploring the roles of integrin binding and cytoskeletal reorganization during mesenchymal stem cell mechanotransduction in soft and stiff hydrogels subjected to dynamic compression. Journal of the Mechanical Behavior of Biomedical Materials (2013), http://dx.doi.org/10.1016/j.jmbbm.2013.07.02 


\section{Introduction}

Both extrinsic and intrinsic mechanical signals (i.e. those generated outside the cell and transmitted inwards via the pericellular matrix (PCM) and those generated within the cell in response to its substrate) have been well characterized as key regulators of mesenchymal stem/stromal cell (MSC) differentiation (Engler et al., 2006; Kelly and Jacobs, 2010; Reilly and Engler, 2010; Steward et al., 2011, 2012, 2013; Thorpe et al., 2012). Dynamic compression (DC) has been shown to enhance chondrogenesis of MSCs depending on the timing and duration of its application (Campbell et al., 2006; Haugh et al., 2011; Huang et al., 2004, 2005, 2010; Mouw et al., 2007; Thorpe et al., 2010); however, the underlying mechanisms responsible for this temporal mechanosensitivity are not well understood. The application of DC from the onset of TGF- $\beta 3$ stimulation has been shown to suppress markers of chondrogenesis, myogenesis and endochondral ossification; however, the application of this mechanical cue following 3 weeks of TGF- $\beta 3$ induced differentiation has been shown to promote chondrogenesis but still suppress markers of both myogenic differentiation (Thorpe et al., 2012) and progression along the endochondral pathway (Thorpe et al., 2013). Both adaption of a chondrogenic phenotype (by long term exposure to chondrogenic growth factors) and development of a mature PCM have been proposed as possible mechanisms to explain the observed temporal mechanosensitivity of MSCs (Haugh et al., 2011). Chondrocytes seeded in agarose hydrogels synthesize a more mature cartilaginous matrix at later time points than at earlier ones, and this more developed matrix may be necessary for the mechanotransduction of DC (Buschmann et al., 1995). Furthermore, blocking integrin-mediated binding to the PCM has been shown to alter chondrocyte response to DC (Kock et al., 2009). A similar temporal development of the PCM has been observed for MSCs embedded into agarose hydrogels (Haugh et al., 2011), however it is still unclear whether this is merely correlating with a more anabolic response to DC or if it is indeed causative.

As the PCM develops not only does its biochemical composition change but it also stiffens (Vigfúsdóttir et al., 2010). The intrinsic stiffness of the local matrix is known to modulate MSC differentiation in both 2D (Engler et al., 2006; Park et al., 2011) and 3D (Huebsch et al., 2010; Murphy et al., 2012; Parekh et al., 2011; Pek et al., 2010). Recently, studies have begun investigating the interplay between extrinsic and intrinsic mechanical cues and their subsequent effects on cellular differentiation. Denser and stiffer hydrogels were found to alter PCM organization, cytoskeletal organization, and integrin binding relative to less dense and softer hydrogels (Steward et al., 2013). These changes led to a more robust response to the application of extrinsic mechanical signals in the stiffer hydrogels, demonstrating the importance of the PCM for determining the response of MSCs to such cues (Steward et al., 2013). These changes to the PCM occurred in parallel with changes to the internal cytoskeleton, raising the possibility that the temporal response of MSCs to extrinsic mechanical cues could be related to changes in either the PCM or to the cytoskeleton during chondrogenesis.

The objective of this study was to first characterize changes in both the pericellular environment and cytoskeletal organization during TGF- $\beta 3$ mediated chondrogenesis of MSCs in hydrogel culture, with the aim of potentially correlating such alterations with the temporal response of the cells to DC. Since both the pericellular environment and cytoskeleton may adapt as differentiation proceeds, decoupling the relative importance of such changes in determining the temporal response of MSCs to DC from other phenotypic changes that may occur in response to increasing exposure to TGF- $\beta 3$ becomes difficult. Therefore we next aimed to determine how altering the pericellular environment of MSCs, by changing the stiffness/ concentration of the hydrogel within which they were encapsulated, would impact their response to DC. Furthermore, we explored how blocking integrin-mediated binding of MSCs to their local pericellular environment would impact their response to DC. The final aim of the study was to elucidate whether cytoskeletal adaptation (i.e. changes in actin, intermediate filaments and microtubules post DC) is a feature of the response of MSCs to extrinsic mechanical loading.

\section{Materials and methods}

\subsection{Cell isolation, expansion, and encapsulation}

Bone marrow was harvested from the femoral diaphysis of 4-month-old pigs ( $\sim 50 \mathrm{~kg})$ under sterile conditions. MSCs were isolated and expanded according to a modified method developed for human MSCs (Lennon and Caplan, 2006). Briefly, bone marrow was removed from the femur, washed and centrifuged twice, and sieved through a $40 \mu \mathrm{m}$ pore-size cell sieve (Falcon, Starstedt). The remaining cell suspension was counted by trypan blue exclusion and seeded at a density of $10 \times 10^{6}$ cells per $175 \mathrm{~cm}^{2}$ T-flask in a humidified atmosphere of $37{ }^{\circ} \mathrm{C}$ and $5 \% \mathrm{CO}_{2}$. Non-adherent cells were removed after 3 days in culture to allow MSCs to attach to the flask. At each passage, cells were reseeded at a density of 875,000 cells per $175 \mathrm{~cm}^{2}$ T-flask. Cultures were expanded in high-glucose Dulbecco's modified Eagle's Medium (hgDMEM GlutaMAX) supplemented with $10 \%$ fetal bovine serum (FBS), and penicillin $(100 \mathrm{U} / \mathrm{mL})$-streptomycin $(100 \mu \mathrm{g} / \mathrm{mL}$ ) (all GIBCO, Biosciences). After expansion (third passage) MSCs were encapsulated in agarose (Type VII, Sigma-Aldrich) at a density of $15 \times 10^{6}$ cells $/ \mathrm{mL}$. Briefly, MSCs were mixed with $5 \%$ agarose at $\sim 40{ }^{\circ} \mathrm{C}$ to yield final gel concentrations of $1 \%$ or $3 \%$ (with equilibrium moduli of 0.5 and $15 \mathrm{MPa}$, respectively). The agarose-cell suspensions were cast in stainless steel molds, and cored using biopsy punches to produce cylindrical scaffolds ( $\varnothing 5 \times 3 \mathrm{~mm}$ thickness). Constructs were maintained in $2.5 \mathrm{~mL} / \mathrm{construct}$ of a chemically defined media (CDM) consisting of hgDMEM GlutaMAX supplemented with penicillin (100 U/mL)-streptomycin (100 $\mu \mathrm{g} / \mathrm{mL})$ (GIBCO, Biosciences), $100 \mu \mathrm{g} / \mathrm{mL}$ sodium pyruvate, $40 \mu \mathrm{g} / \mathrm{mL}$ L-proline, $50 \mu \mathrm{g} / \mathrm{mL}$ L-ascorbic acid-2-phosphate, $1.5 \mathrm{mg} / \mathrm{mL}$ BSA, $1 \times$ insulintransferrin-selenium, $100 \mathrm{nM}$ dexamethasone (all SigmaAldrich), and $10 \mathrm{ng} / \mathrm{mL}$ recombinant human transforming growth factor- $\beta 3$ (chondrogenic, TGF- $\beta 3$; ProSpec-Tany TechnoGene Ltd). Additional groups were also cultured with the addition of a $167 \mu \mathrm{M}$ RGDS peptide (RGDS+, Tocris Bioscience) in order to inhibit integrin binding during the first week of culture. Cells that were to be cultured with the RGDS peptide were equilibrated in the chondrogenic media supplemented 
with RGDS for $2 \mathrm{~h}$ prior to encapsulation in agarose, and RGDS was added to the media during each subsequent media change.

\subsection{Application of dynamic compression}

Constructs were placed in a custom pneumatic based compressive loading bioreactor (Thorpe et al., 2010) inside an incubator $\left(37^{\circ} \mathrm{C}, 5 \% \mathrm{CO}_{2}\right)$ and exposed to cyclic dynamic compression (DC) on days 7, 14, or 21 only. Constructs were not loaded on any other day. The $1 \%$ constructs were only loaded on day 7. DC was applied with $\sim 10 \%$ strain superimposed on a $0.01 \mathrm{~N}$ preload at a frequency of $1 \mathrm{~Hz}$ for $1 \mathrm{~h}$ only. Free swelling (FS) controls were kept in the incubator adjacent to the bioreactor. Half-medium exchanges were performed every 3-4 days.

\subsection{Confocal microscopy and histology}

Immediately after the given loading regime (days 7, 14, or 21), constructs $(n=2)$ were cut in half and fixed in $4 \%$ paraformaldehyde (Sigma-Aldrich) overnight at $4{ }^{\circ} \mathrm{C}$ and rinsed with PBS. In order to examine focal adhesion formation and cytoskeletal organization, samples were permeabilized in a $1 \%$ Triton-X and 2\% BSA solution for $45 \mathrm{~min}$ and washed in PBS. The samples were then incubated in a $1.5 \%$ BSA solution containing one of either $60 \mu \mathrm{g} / \mathrm{mL}$ monoclonal anti-vinculin FITC conjugate (Sigma-Aldrich), $5 \mathrm{U} / \mathrm{mL}$ rhodamine phalloidin (VWR International, Ireland), $1 \mu \mathrm{g} / \mathrm{mL}$ anti-vimentin FITC (eBioscience, Inc.), or $1 \mu \mathrm{g} / \mathrm{mL}$ anti-alpha tubulin eFluor ${ }^{\circledR} 615$ (eBioscience, Inc.) for $1.5 \mathrm{~h}$, and then imaged using a Zeiss 510 Meta confocal microscope at $40 \times$ magnification.

The remaining halves were dehydrated and embedded in paraffin wax. Constructs were sectioned perpendicular to the disc face yielding $5 \mu \mathrm{m}$ thick sections. Sections were stained with either 1\% Alcian blue 8GX (Sigma-Aldrich, Ireland) in $0.1 \mathrm{M} \mathrm{HCl}$ for sGAG, or picro-sirius red to detect collagen.

\subsection{RNA isolation and quantitative real-time polymerase chain reaction}

Quantitative real-time reverse transcription polymerase chain reaction (qRT-PCR) was used to determine relative gene expression changes in chondrogenic specific genes with respect to both application of loading and supplementation with RGDS peptide. Total RNA was extracted from agarose constructs directly after loading on day 7, 14, or 21 of culture. Total RNA was extracted by homogenizing each construct with an Ultraturrax IKA T10 basic homogenizer (Fisher Scientific) in $1 \mathrm{~mL}$ of TRIZOL reagent (Invitrogen), followed by a chloroform (SigmaAldrich) extraction. The extracted solution was incubated with an equal volume of isopropanol and $5 \mu \mathrm{L}$ glycogen in a $-20{ }^{\circ} \mathrm{C}$ freezer overnight. The solution was then centrifuged and the precipitate was washed once with $70 \%$ ethanol. The precipitate was resuspended in $200 \mu \mathrm{L}$ of $35 \%$ ethanol and the RNA was then extracted with a PureLink ${ }^{\mathrm{TM}}$ RNA Mini kit (Invitrogen) as per manufacturer's instructions. Total RNA yield and purity were analyzed using an ND 1000 NanoDrop Spectrophotometer (Labtech International) and adjusted to a standard concentration prior to cDNA synthesis. To quantify mRNA expression,
50 ng of total RNA was reverse transcribed into cDNA using a high capacity reverse transcription cDNA kit (Applied Biosystems) as per manufacturer's instructions. TaqMan ${ }^{\circledR}$ gene expression assays (Applied Biosystems) which contain forward and reverse primers, and a FAM-labeled TaqMan probe for porcine Sox9 (Ss03392406_m1), aggrecan (Agc, Ss03374822_m1), collagen type II alpha 1 (Col2A1, Ss03373344_g1), transforming growth factor beta receptor 1 (TGFBR1, Ss03392141_m1), transforming growth factor beta 3 (TGB3, Ss03394351_m1), bone morphogenetic protein 2 (BMP2, Ss03373798_g1), bone morphogenetic protein 7 (BMP7, Ss03389523_m1), and glyceraldehydethree-phosphate dehydrogenase (GAPDH, Ss03373286) were used in this study. qRT-PCR was performed using an ABI 7500 Sequence Detection system (Applied Biosystems). A volume of $5 \mu \mathrm{L}$ of cDNA preparation (diluted 1:5 with RNase free water), $1 \mu \mathrm{L}$ of gene assay, $10 \mu \mathrm{L}$ TaqMan Universal PCR Master mix (Applied Biosystems) and $4 \mu \mathrm{L}$ RNase free water (20 $\mu \mathrm{L}$ total volume) were added to each well. Samples were assayed in triplicate in one run (40 cycles). qRT-PCR data were analyzed using the $\Delta \Delta \mathrm{C}_{\mathrm{T}}$ method as described previously (Livak and Schmittgen, 2001) with GAPDH as the endogenous control. Relative quantification values are presented as fold changes in gene expression relative to the control group.

\subsection{Statistical analysis}

Statistical analysis was performed using GraphPad Prism (version 5.00, GraphPad Software) with a minimum of three samples in triplicate analyzed for each experimental group for each assay. qRT-PCR results, both numerical and graphical, are expressed in the form of mean \pm standard deviation. Differences between DC and FS samples or between samples cultured with or without RGDS were determined using a Student's t-test. A level of $p<0.05$ was considered significant.

\section{3. $\quad$ Results}

\subsection{MSCs display a temporal response to dynamic} compression despite minimal changes to their gross actin, vimentin and tubulin networks during chondrogenesis in $3 \mathrm{D}$ hydrogels

MSCs embedded into stiff 3\% agarose hydrogels were subjected to $1 \mathrm{~h}$ (total duration) of dynamic compression (DC) following either 7, 14 or 21 days of free swelling (FS) culture in chondrogenic media. Similar to previous results (Haugh et al., 2011), MSCs displayed a temporal gene expression pattern in response to DC (Fig. 1A), with a more anabolic response observed on day 21 compared to day 14. Sox9 expression was significantly increased by DC (relative to FS) on day $7(1.63 \pm 0.40)$, decreased on day $14(0.21 \pm 0.59)$, with no significant change on day 21 (0.74 \pm 1.33$)$. DC had no significant effect on Agc expression on day 7 (1.27 \pm 0.37$)$, but significantly enhanced Agc gene expression on days 14 and 21 ( $2.39 \pm 1.03$ and $1.85 \pm 0.39$, respectively). DC significantly increased Col2A1 expression on days 7 and 21 $(6.58 \pm 1.36$ and $2.97 \pm 0.86$, respectively), while it significantly decreased its expression on day $14(0.35 \pm 0.51)$. Next, the PCM and cytoskeleton were analyzed in order to determine if 

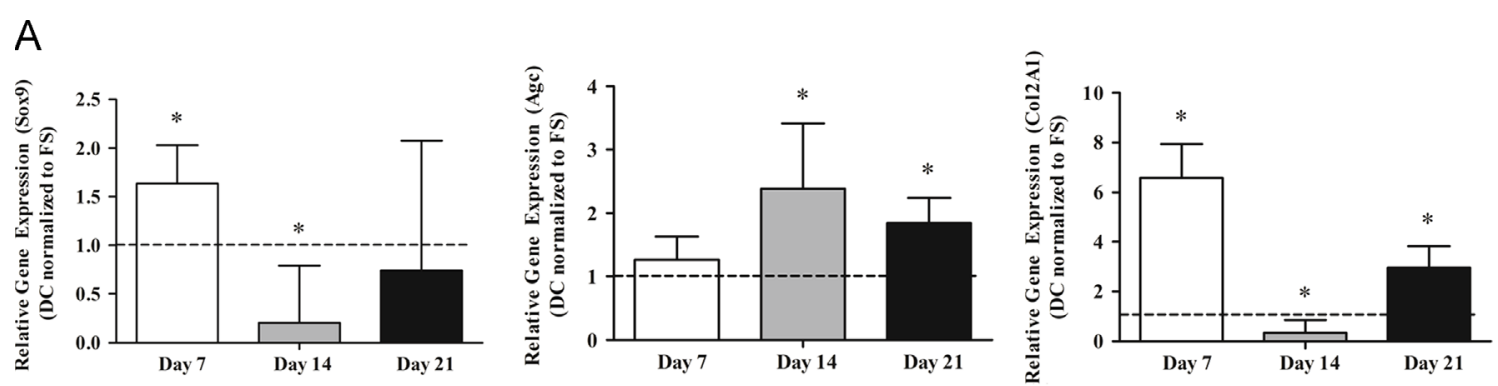

B

Day 7

Day 14
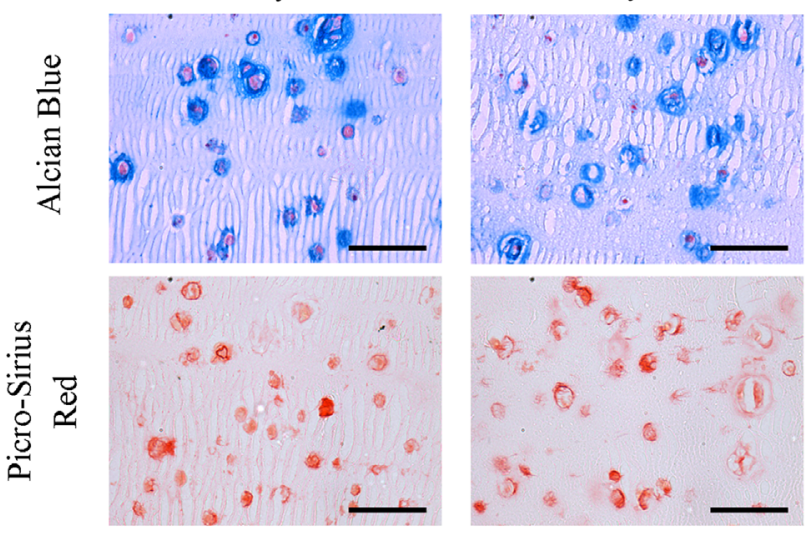

Day 21

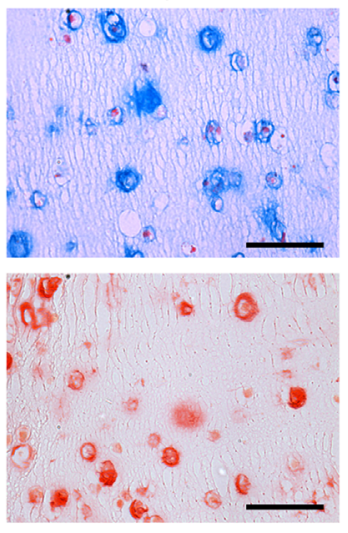

C
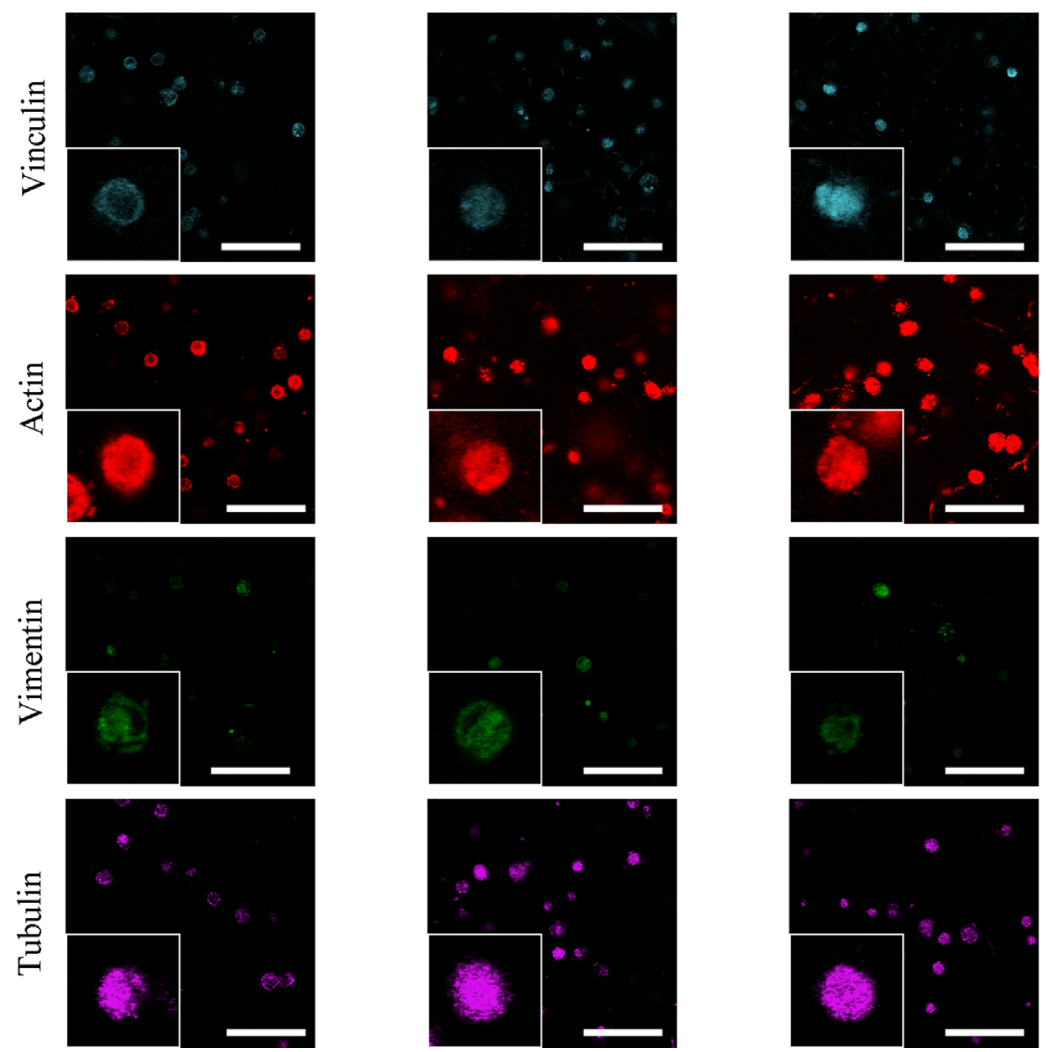

Fig. 1 - (A) Sox9, Agc, and Col2A1 gene expression in stiff hydrogels normalized to FS condition. (B) Representative Alcian blue and picro-sirius red images, and (C) representative vinculin, actin, vimentin and tubulin confocal images of stiff hydrogels on days 7, 14, and 21 (scale bar $=50 \mu \mathrm{m} ; \mathbf{1 2 . 5} \mu \mathrm{m}$ for inset images). ${ }^{*} p<0.05$ relative to FS. (For interpretation of the references to color in this figure legend, the reader is referred to the web version of this article.)

temporal changes in their development and organization correlated with the observed temporal effects of DC on MSC chondrogenic gene expression. The PCM of MSCs stained intensely for
Alcian blue and picro-sirius red, but no dramatic changes were observed with time in culture (Fig. 1B), suggesting the PCM develops rapidly in the stiff/dense 3\% agarose hydrogels. All of

Please cite this article as: Steward, A.J., et al., Exploring the roles of integrin binding and cytoskeletal reorganization during mesenchymal stem cell mechanotransduction in soft and stiff hydrogels subjected to dynamic compression. Journal of the Mechanical Behavior of Biomedical Materials (2013), http://dx.doi.org/10.1016/j.jmbbm.2013.07.020 
the cytoskeletal elements (actin, vimentin, and tubulin) generally exhibited a diffuse staining within spherical cells, with minimal changes observed in the gross appearance of these elements with time in culture (Fig. 1C). Vinculin (a membrane-cytoskeletal protein in focal adhesions) staining appeared more intense by day 21 (Fig. 1C).

\subsection{A well developed PCM appears important for dynamic} compression to promote chondrogenesis of MSCs

Given that relatively minor changes in both the gross cytoskeleton and PCM were observed with time in culture, we next explored how modulating the development of the PCM (by reducing the agarose hydrogel concentration from $3 \%$ to $1 \%$ ) would impact the response of MSCs to DC. The total duration of exposure to TGF- $\beta 3$ was kept constant (7 days) in order to minimise other changes in phenotype that will occur within the hydrogels with increasing duration of exposure to this growth factor. The application of DC on day 7 elicited a significantly greater relative increase in Sox9 (soft: $0.70 \pm 0.15$, stiff: $1.63 \pm 0.40$ ), Agc (soft: $0.47 \pm 0.28$, stiff: $1.26 \pm 0.37$ ) and Col2A1 (soft: $1.77 \pm 0.13$, stiff: $6.58 \pm 1.36$ ) gene expression in MSCs embedded in stiff hydrogels compared to soft hydrogels. The PCM was less developed in the soft 1\% agarose hydrogels compared to the stiffer 3\% gels (Fig. 2B), implicating a well developed PCM in the mechanotransduction of DC into an anabolic change in chondrogenic gene expression. Again, all cytoskeletal elements exhibited a generally diffuse staining, with little differences observed in the gross cytoskeletal structure between the soft and stiff hydrogels (Fig. 2C).
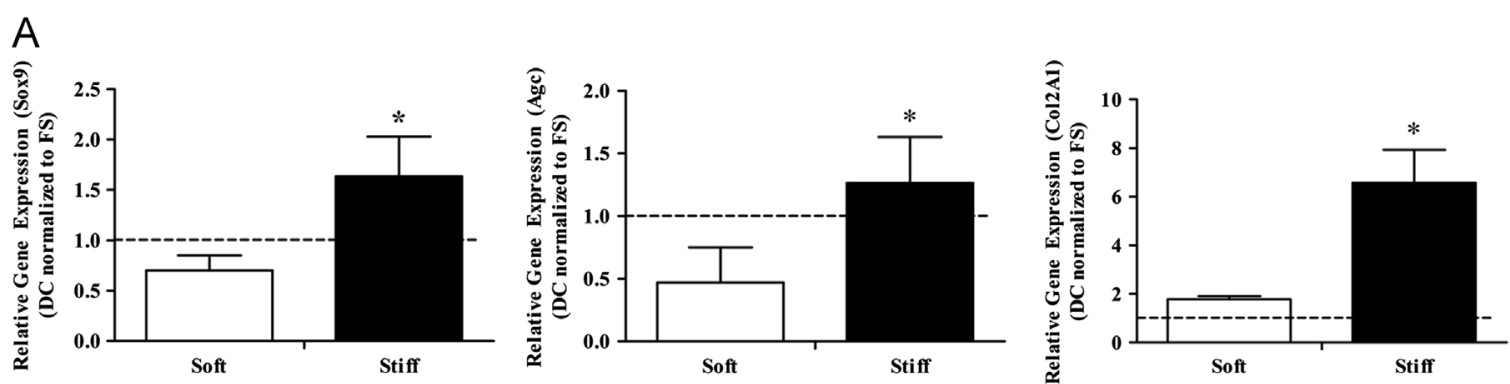

B
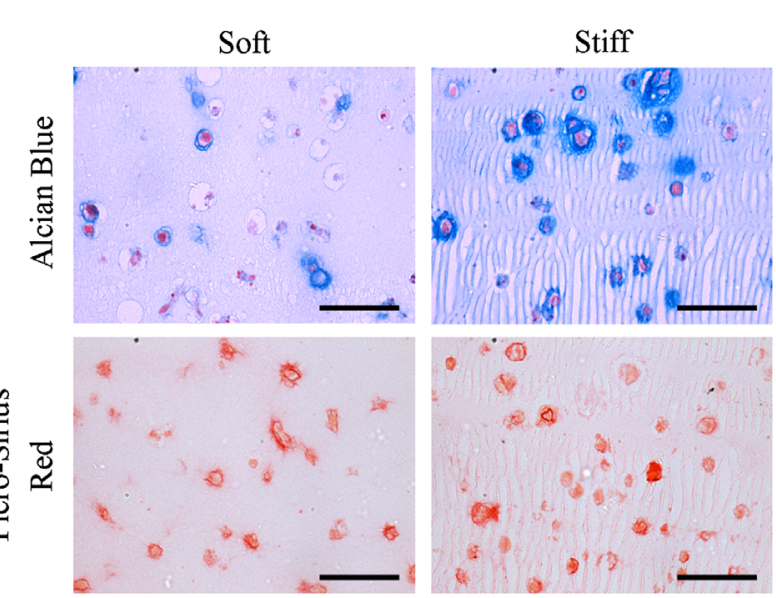

C
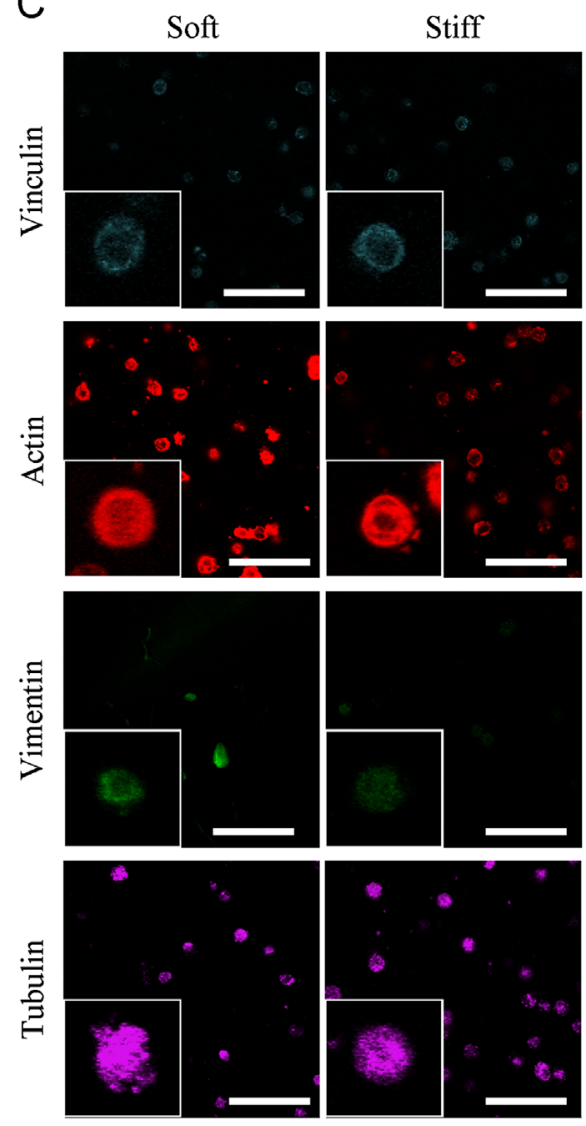

Fig. 2 - (A) Sox9, Agc, and Col2A1 gene expression in soft and stiff hydrogels normalized to FS condition on day 7.

(B) Representative Alcian blue and picro-sirius red images, and (C) representative vinculin, actin, vimentin and tubulin confocal images of soft and stiff hydrogels on days 7 (scale bar $=50 \mu \mathrm{m} ; \mathbf{1 2 . 5} \mu \mathrm{m}$ for inset images). * $p<0.05$ relative to soft hydrogels. (For interpretation of the references to color in this figure legend, the reader is referred to the web version of this article.)

Please cite this article as: Steward, A.J., et al., Exploring the roles of integrin binding and cytoskeletal reorganization during mesenchymal stem cell mechanotransduction in soft and stiff hydrogels subjected to dynamic compression. Journal of the Mechanical Behavior of Biomedical Materials (2013), http://dx.doi.org/10.1016/j.jmbbm.2013.07.020 


\subsection{Integrin mediated binding by MSCs to their PCM is} involved in mechanotransduction of dynamic compression

Next, to determine if integrin-mediated binding of MSCs to their local PCM was necessary for mechanotransduction of DC, integrin binding was inhibited in the stiffer $3 \%$ gels, where an anabolic response to DC was observed, with addition of the soluble RGDS peptide for the first 7 days of culture. The significant increase in Sox9 and Col 2A1 gene expression in response to DC was not observed with the addition of RGDS (Sox9: 1.63 $\pm 0.40,0.56 \pm 0.20$; Col2A1: $6.58 \pm 1.36,1.49 \pm 0.11$ ). The addition of RGDS had no significant effect on Agc expression after application of DC (Fig. 3A). No differences in PCM development or gross cytoskeletal organization were observed with the addition of RGDS (Fig. 3B and C).
3.4. Tubulin rapidly reorganises within the cytoskeleton of MSCs in response to DC

Although there were no temporal changes observed in cytoskeletal structure, we next examined if either the actin, vimentin or tubulin networks in MSCs were altered by the application of DC in the stiff 3\% hydrogels, where an anabolic response to loading was observed. DC induced no observable changes in gross actin or vimentin structure in MSCs seeded in stiff $3 \%$ hydrogels (data not shown). However, tubulin displayed an altered ring morphology after the application of DC, appearing to localise around the cell membrane and nucleus following loading (Fig. 4). Interestingly, this effect was not negated by the addition of RGDS, as microtubule organization still adapted to DC even in the presence of the RGDS peptide (Fig. 4).
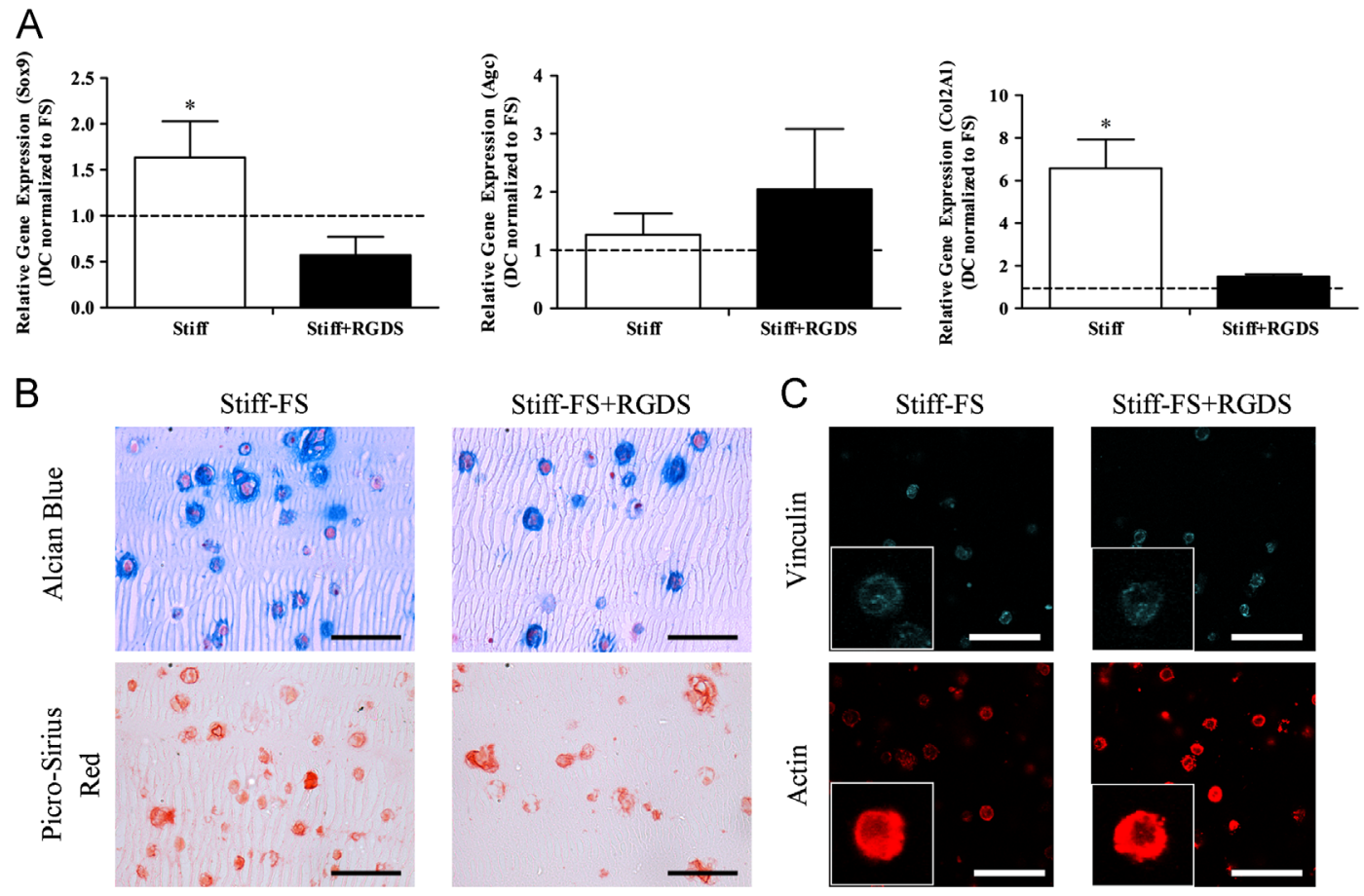

Stiff-FS+RGDS
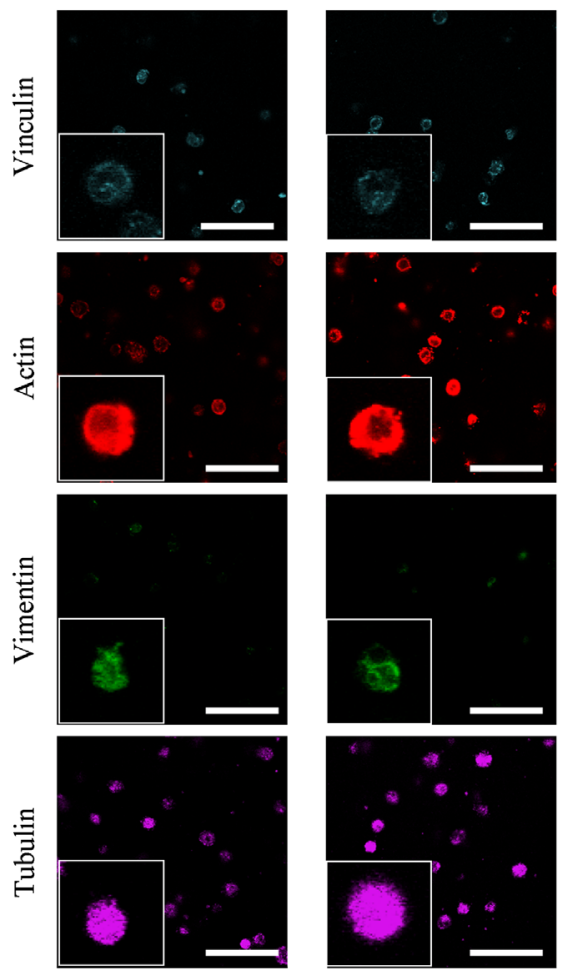

Fig. 3 - (A) Sox9, Agc, and Col2A1 gene expression in stiff hydrogels on day 7, with or without the RGDS peptide, normalized to FS condition. (B) Representative Alcian blue and picro-sirius red images, and (C) representative vinculin, actin, vimentin and tubulin confocal images of stiff hydrogels on day 7, with or without the RGDS peptide (scale bar $=50 \mu \mathrm{m}$; $12.5 \mu \mathrm{m}$ for inset images). * $p<0.05$ relative to groups cultured with RGDS peptide. (For interpretation of the references to color in this figure legend, the reader is referred to the web version of this article.)

Please cite this article as: Steward, A.J., et al., Exploring the roles of integrin binding and cytoskeletal reorganization during mesenchymal stem cell mechanotransduction in soft and stiff hydrogels subjected to dynamic compression. Journal of the Mechanical Behavior of Biomedical Materials (2013), http://dx.doi.org/10.1016/j.jmbbm.2013.07.02 

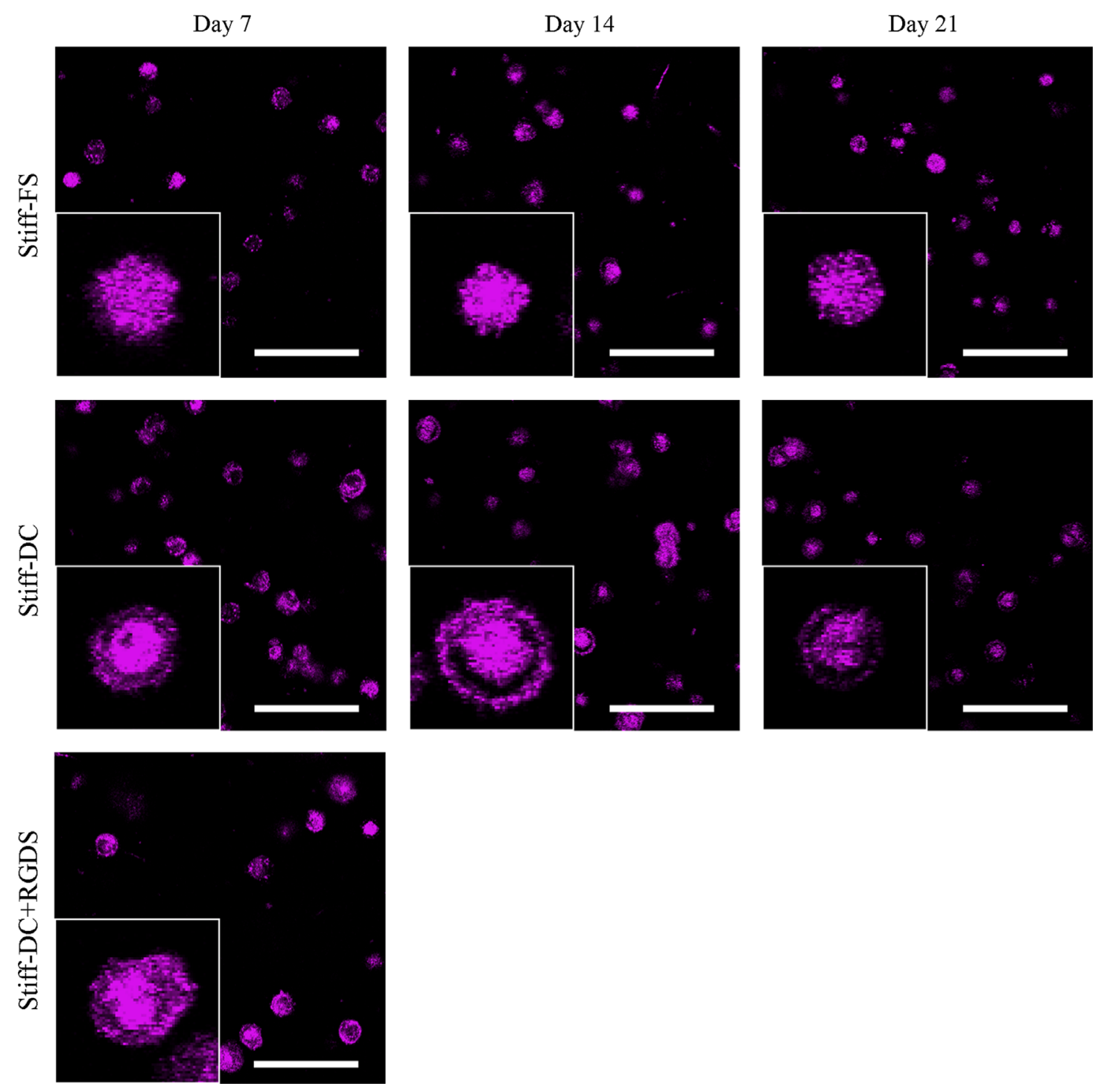

Fig. 4 - Representative tubulin confocal images on days 7, 14, and 21 in either the FS, DC, or DC with RGDS conditions (scale bar $=50 \mu \mathrm{m} ; 12.5 \mu \mathrm{m}$ for inset images).

\section{Discussion}

Numerous studies have demonstrated that MSCs display a temporal response to the application of dynamic compression (Haugh et al., 2011; Huang et al., 2004, 2005, 2010; Mouw et al., 2007; Thorpe et al., 2010). A similar result was observed in the present study. The cytoskeleton has often been proposed to play a key role in mechanotransduction and MSC differentiation (Connelly et al., 2008; Discher et al., 2005; McBeath et al., 2004; Steward et al., 2012). Therefore we initially hypothesised that cytoskeletal development may explain the temporal gene expression patterns observed in MSCs in response to DC. However, no dramatic differences were observed in the gross cytoskeletal organization over time or in how the cytoskeleton adapted to loading over time, suggesting that changes to the cytoskeleton (that can be detected using the experimental approach adopted in this study) during chondrogenesis are not responsible for the temporal gene expression profile observed in MSCs in response to DC.

Next, the possible role of PCM development in determining the response of MSCs to DC was examined. No dramatic differences in PCM maturation were observed between day 7 and day 21 of culture in the stiff/dense 3\% agarose hydrogels (where the PCM develops relatively quickly), initially suggesting that PCM maturation does not play a role in determining the temporal response of MSCs to loading. Previous work in our lab has shown that the PCM develops differently in agarose gels of different concentrations, with the PCM becoming more developed and compact in stiffer hydrogels (Steward et al., 2013). Therefore, to further probe the role of the pericellular environment in MSC mechanotransduction, 'soft' and 'stiff' MSC seeded agarose gels were subjected to DC after 7 days in chondrogenic media. A less well developed PCM was observed in the softer $1 \%$ gels, which correlated with a less anabolic response to DC than that observed in the stiffer $3 \%$ gels. It is well established that the PCM plays an

Please cite this article as: Steward, A.J., et al., Exploring the roles of integrin binding and cytoskeletal reorganization during mesenchymal stem cell mechanotransduction in soft and stiff hydrogels subjected to dynamic compression. Journal of the Mechanical Behavior of Biomedical Materials (2013), http://dx.doi.org/10.1016/j.jmbbm.2013.07.020 
important role in chondrocyte biomechanics and mechanotransduction (Alexopoulos et al., 2005; Buschmann et al., 1995; Choi et al., 2007; Guilak et al., 2006). Finite element models have been used to demonstrate that the presence of a PCM dramatically alters the mechanical environment of cells that are embedded in hydrogels subjected to DC, decreasing the deformation and deviatoric strains experienced by the cell but increasing the volumetric strain (Khoshgoftar et al., 2013), which may be important for appropriate mechanotransduction. Taken together, these findings suggest that a well developed PCM is important to ensure an anabolic response to DC, although changes to the PCM may not completely explain the temporal gene expression patterns observed in response to extrinsic loading in this and other studies.

While it is well established that both intrinsic and extrinsic mechanical signals drive differentiation of MSCs, the mechanotransductive pathways with which MSCs respond to these signals are not fully understood. Integrin binding to the extracellular matrix (ECM) and cytoskeletal organization have long been implicated in many mechanotransductive pathways (Ingber, 2007; Wang et al., 1993, 2009). The integrincytoskeletal linkage becomes stronger with increasing matrix stiffness, suggesting a potential downstream role for cytoskeletal organization in mechanotransduction (Choquet et al., 1997). Given that PCM maturation was critical for mechanotransduction of DC, and that previous work has demonstrated that integrin binding to the PCM was needed for mechanotransduction of cyclic hydrostatic pressure to occur (Steward et al., 2013), we next explored whether integrin binding was also necessary for proper mechanotransduction of DC by abrogating integrin mediated binding with a soluble RGDS peptide. Similar to MSC response to hydrostatic pressure in the presence of RGDS (Steward et al., 2013), no significant increase in Sox9 and Col2A1 gene expression in response to DC was observed in the presence of RGDS. This suggests that integrin mediated binding to the PCM plays a key role in the mechanotransduction of DC into a prochondrogenic signal to the cell.

Previously, the cytoskeleton was found to remodel in MSCs exposed to hydrostatic pressure, and this remodelling was inhibited in the presence of RGDS (Steward et al., 2013). In the current study, it was found that the tubulin network in MSCs remodelled in response to DC. Microtubules have previously been demonstrated to play a role in resisting cell deformation (Ingber, 1993, 1997; Mizushima-Sugano et al., 1983), and together with this study, suggests that microtubule reorganization plays a key role in the response and adaption of chondrogenically primed MSCs to DC. Interestingly, the addition of RGDS did not inhibit tubulin reorganization, suggesting that integrin mediated binding to the PCM is not involved in tubulin reorganization in response to DC. Since microtubules appear to adapt in both the presence and absence of RGDS, but that DC only enhanced chondrogenic gene expression in the absence of RGDS, tubulin reorganization in of itself may not be involved in the mechanotransduction of DC into an anabolic change in chondrogenic gene expression.

In conclusion, MSCs embedded in agarose hydrogels were found to display a temporal chondrogenic gene expression pattern in response to DC. Neither changes in gross cytoskeletal organization nor PCM development could explain these temporal gene expression patterns, although it is acknowledged that certain changes may be occurring that are not detectable using the experimental methods adopted in this study. We did however observe greater increases in the relative expression of chondrogenic marker genes in response to DC in the presence of a well developed PCM. Integrin-mediated binding to the PCM was also observed to play a key role in mechanotransduction of DC. Microtubules were found to reorganize in MSCs exposed to DC, demonstrating that the microtubule network in chondrogenically primed MSCs is mechanosensitive; however, this network does not appear to be involved in integrin binding-mediated mechanotransduction of DC into increases in Sox9 and Col2A1 gene expression as microtubule reorganization in response to DC was not inhibited by abrogating integrin binding. Therefore, although the PCM, integrin binding, and cytoskeletal organization are all involved in MSC mechanotransduction, none of these factors in isolation can provide a clear explanation for the temporal response of MSCS to DC.

\section{Acknowledgements}

This study was supported by a President of Ireland Young Researcher Award from Science Foundation Ireland (08/Y15/ B1336), funding from a European Research Council Starter Grant (No. 258463) and a Naughton Graduate Fellowship.

\section{R E F E R E C E S}

Alexopoulos, L.G., Setton, L.A., Guilak, F., 2005. The biomechanical role of the chondrocyte pericellular matrix in articular cartilage. Acta Biomaterialia 1, 317-325.

Buschmann, M.D., Gluzband, Y.A., Grodzinsky, A.J., Hunziker, E.B., 1995. Mechanical compression modulates matrix biosynthesis in chondrocyte/agarose culture. Journal of Cell Science 108, 1497-1508.

Campbell, J.J., Lee, D.A., Bader, D.L., 2006. Dynamic compressive strain influences chondrogenic gene expression in human mesenchymal stem cells. Biorheology 43, 455-470.

Choi, J.B., Youn, I., Cao, L., Leddy, H.A., Gilchrist, C.L., Setton, L.A., Guilak, F., 2007. Zonal changes in the three-dimensional morphology of the chondron under compression: the relationship among cellular, pericellular, and extracellular deformation in articular cartilage. Journal of Biomechanics 40, 2596-2603.

Choquet, D., Felsenfeld, D.P., Sheetz, M.P., 1997. Extracellular matrix rigidity causes strengthening of integrin-cytoskeleton linkages. Cell 88, 39-48.

Connelly, J.T., Garcia, A.J., Levenston, M.E., 2008. Interactions between integrin ligand density and cytoskeletal integrity regulate BMSC chondrogenesis. Journal of Cellular Physiology, 217.

Discher, D.E., Janmey, P., Wang, Y., 2005. Tissue cells feel and respond to the stiffness of their substrate. Science 310, 1139-1143.

Engler, A.J., Sen, S., Sweeney, H.L., Discher, D.E., 2006. Matrix elasticity directs stem cell lineage specification. Cell 126, 677-689.

Guilak, F., Alexopoulos, L.G., Upton, M.L., Youn, I., Choi, J.B., Cao, L., Setton, L.A., Haider, M.A., 2006. The pericellular matrix as a transducer of biomechanical and biochemical signals in 
articular cartilage. Annals of the New York Academy of Sciences 1068, 498-512.

Haugh, M.G., Meyer, E.G., Thorpe, S.D., Vinardell, T., Duffy, G.P., Kelly, D.J., 2011. Temporal and spatial changes in cartilagematrix-specific gene expression in mesenchymal stem cells in response to dynamic compression. Tissue Engineering Part A (110826123433006).

Huang, A.H., Farrell, M.J., Kim, M., Mauck, R.L., 2010. Long-term dynamic loading improves the mechanical properties of chondrogenic mesenchymal stem cell-laden hydrogel. European Cells and Materials 19, 72-85.

Huang, C.C., Hagar, K.L., Frost, L.E., Sun, Y., Cheung, H.S., 2004 Effects of cyclic compressive loading on chondrogenesis of rabbit bone-marrow derived mesenchymal stem cells. Stem Cells 22, 313-323.

Huang, C.Y.C., Reuben, P.M., Cheung, H.S., 2005. Temporal expression patterns and corresponding protein inductions of early responsive genes in rabbit bone marrow-derived mesenchymal stem cells under cyclic compressive loading. Stem Cells 23, 1113-1121.

Huebsch, N., Arany, P.R., Mao, A.S., Shvartsman, D., Ali, O.A., Bencherif, S.A., Rivera-Feliciano, J., Mooney, D.J., 2010. Harnessing traction-mediated manipulation of the cell/matrix interface to control stem-cell fate. Nature Materials 9, 518-526.

Ingber, D.E., 1993. Cellular tensegrity: defining new rules of biological design that govern the cytoskeleton. Journal of Cell Science 104, 613-627.

Ingber, D.E., 1997. Tensegrity: the architectural basis of cellular mechanotransduction. Annual Review of Physiology 59, 575.

Ingber, D.E., 2007. Integrins, tensegrity, and mechanotransduction. Gravitational and Space Biology, 10.

Kelly, D.J., Jacobs, C.R., 2010. The role of mechanical signals in regulating chondrogenesis and osteogenesis of mesenchymal stem cells. Birth Defects Research Part C: Embryo Today: Review 90, 75-85.

Khoshgoftar, M., Wilson, W., Ito, K., van Donkelaar, R., 2013. The effects of matrix inhomogeneities on the cellular mechanical environment in tissue-engineered cartilage: an in silico investigation. Tissue Engineering Part C: Methods (130516192052006).

Kock, L.M., Schulz, R.M., van Donkelaar, C.C., Thümmler, C.B., Bader, A., Ito, K., 2009. RGD-dependent integrins are mechanotransducers in dynamically compressed tissueengineered cartilage constructs. Journal of Biomechanics 42, $2177-2182$.

Lennon, D.P., Caplan, A.I., 2006. Isolation of human marrowderived mesenchymal stem cells. Experimental Hematology 34, 1604-1605.

Livak, K.J., Schmittgen, T.D., 2001. Analysis of relative gene expression data using real-time quantitative PCR and the 2$\Delta \Delta \mathrm{C}_{\mathrm{T}}$ method. Methods 25, 402-408.

McBeath, R., Pirone, D.M., Nelson, C.M., Bhadriraju, K., Chen, C.S., 2004. Cell shape, cytoskeletal tension, and RhoA regulate stem cell lineage commitment. Developmental Cell 6, 483-495.

Mizushima-Sugano, J., Maeda, T., Miki-Noumura, T., 1983. Flexural rigidity of singlet microtubules estimated from statistical analysis of their contour lengths and end-to-end distances. Biochimica et Biophysica Acta 755, 257-262.

Mouw, J.K., Connelly, J.T., Wilson, C.G., Michael, K.E., Levenston, M.E., 2007. Dynamic compression regulates the expression and synthesis of chondrocyte-specific matrix molecules in bone marrow stromal cells. Stem Cells 25, 655-663.

Murphy, C.M., Matsiko, A., Haugh, M.G., Gleeson, J.P., O'Brien, F.J., 2012. Mesenchymal stem cell fate is regulated by the composition and mechanical properties of collagenglycosaminoglycan scaffolds. Journal of the Mechanical Behavior of Biomedical Materials 11, 53-62.

Parekh, S.H., Chatterjee, K., Lin-Gibson, S., Moore, N.M., Cicerone, M.T., Young, M.F., Simon Jr., C.G., 2011. Modulus-driven differentiation of marrow stromal cells in 3D scaffolds that is independent of myosin-based cytoskeletal tension. Biomaterials 32, 2256-2264.

Park, J.S., Chu, J.S., Tsou, A.D., Diop, R., Tang, Z., Wang, A., Li, S., 2011. The effect of matrix stiffness on the differentiation of mesenchymal stem cells in response to TGF-[beta]. Biomaterials 32, 3921-3930.

Pek, Y.S., Wan, A.C.A., Ying, J.Y., 2010. The effect of matrix stiffness on mesenchymal stem cell differentiation in a 3D thixotropic gel. Biomaterials 31, 385-391.

Reilly, G.C., Engler, A.J., 2010. Intrinsic extracellular matrix properties regulate stem cell differentiation. Journal of Biomechanics 43, 55-62.

Steward, A.J., Liu, Y., Wagner, D.R., 2011. Engineering cell attachments to scaffolds in cartilage tissue engineering. Journal of Operations Management 63, 74-82.

Steward, A.J., Thorpe, S.D., Vinardell, T., Buckley, C.T., Wagner, D.R., Kelly, D.J., 2012. Cell-matrix interactions regulate mesenchymal stem cell response to hydrostatic pressure. Acta Biomaterialia 8, 2153-2159.

Steward, A.J., Wagner, D.R., Kelly, D.J., 2013. The pericellular environment regulates cytoskeletal development and the differentiation of mesenchymal stem cells and determines their response to hydrostatic pressure. European Cells and Materials 25, 167-178.

Thorpe, S.D., Buckley, C.T., Steward, A.J., Kelly, D.J., 2012. European society of biomechanics S.M. Perren Award 2012: the external mechanical environment can override the influence of local substrate in determining stem cell fate. Journal of Biomechanics 45, 2483-2492.

Thorpe, S.D., Buckley, C.T., Vinardell, T., O’Brien, F.J., Campbell, V.A. Kelly, D.J., 2010. The response of bone marrow-derived mesenchymal stem cells to dynamic compression following TGF- $\beta 3$ induced chondrogenic differentiation. Annals of Biomedical Engineering 38, 2896-2909.

Thorpe, S.D., Nagel, T., Carroll, S.F., Kelly, D.J., 2013. Modulating gradients in regulatory signals within mesenchymal stem cell seeded hydrogels: a novel strategy to engineer zonal articular cartilage. PLoS One 8, e60764.

Vigúsdóttir, Á.T., Pasrija, C., Thakore, P.I., Schmidt, R.B., Hsieh, A.H., 2010. Role of pericellular matrix in mesenchymal stem cell deformation during chondrogenic differentiation. Cellular and Molecular Bioengineering 3, 387-397.

Wang, N., Butler, J., Ingber, D., 1993. Mechanotransduction across the cell surface and through the cytoskeleton. Science 260, 1124-1127.

Wang, N., Tytell, J.D., Ingber, D.E., 2009. Mechanotransduction at a distance: mechanically coupling the extracellular matrix with the nucleus. Nature Reviews Molecular Cell Biology 10, 75-82. 